\title{
REPRESENTATION OF MARTINGALES, QUADRATIC VARIATION AND APPLICATIONS*
}

\author{
E. WONG $\dagger$
}

\begin{abstract}
In this paper, we present two related results. First, we shall obtain a sufficient condition under which a second order sample-continuous martingale can be represented as a stochastic integral in terms of a Brownian motion. Secondly, we shall show that if $X$ and $Y$ are sample-continuous local martingales (not necessarily with respect to the same family of $\sigma$-algebras) and if either $X+Y$ or $X-Y$ is almost surely of bounded variation, then the quadratic variations of the two martingales are equal. This rather simple result has some surprising consequences.
\end{abstract}

1. Introduction. Let $\left\{X_{t}, t \geqq 0\right\}$ be a sample-continuous second order martingale. Then $\left\{X_{t}^{2}, t \geqq 0\right\}$ is a sample-continuous first order submartingale and the conditions for Meyer's decomposition [1] are always satisfied; thus we can write

$$
X_{t}^{2}=M_{t}+A_{t}, \quad t \geqq 0,
$$

where $M$ is a martingale, $A$ is an increasing process, and both are sample-continuous. The decomposition is unique if we set $M_{0}=X_{0}^{2}$. If $X$ is a local martingale [2], (1) remains valid except now $M$ is a local martingale. Following Kunita and Watanabe [2], we shall adopt the suggestive notation $\langle X\rangle_{t}$ for the increasing process $A_{t}$.

In this paper, we present two related results. First, we shall obtain a sufficient condition under which a second order sample-continuous martingale can be represented as a stochastic integral in terms of a Brownian motion. Second, we shall show that if $X$ and $Y$ are sample-continuous local martingales (not necessarily with respect to the same family of $\sigma$-algebras) and if either $X+Y$ or $X-Y$ is almost surely of bounded variation, then $\langle X\rangle_{t}=\langle Y\rangle_{t}$. This rather simple result has some surprising consequences.

2. Martingales and stochastic integrals. Let $(\Omega, \mathscr{A}, \mathscr{P})$ be a probability space, and let $\left\{\mathscr{A}_{t}, t \geqq 0\right\}$ be an increasing family of sub- $\sigma$-algebras. A process $\left\{X_{t}, t \geqq 0\right\}$ is said to be adapted to $\left\{\mathscr{A}_{t}\right\}$ if, for each $t, X_{t}$ is $\mathscr{A}_{t}$-measurable. We say that $\left\{X_{t}, \mathscr{A}_{t}\right\}$ is a martingale if $X$ is adapted to $\left\{\mathscr{A}_{t}\right\}$ and for every $t>s$,

$$
E^{\mathscr{A}_{s}} X_{t}=X_{s}
$$

almost surely. If $\left\{X_{t}, \mathscr{A}_{t}\right\}$ is a sample-continuous second order martingale, then the increasing process $\langle X\rangle_{t}$ introduced earlier is well-defined and $E\langle X\rangle_{t}<\infty$.

A nonnegative random variable $\tau$ is said to be a stopping time of $\left\{\mathscr{A}_{t}\right\}$ if $\{\omega: \tau(\omega) \leqq t\} \in \mathscr{A}_{t}$ for every $t$. A process $\left\{X_{t}, \mathscr{A}_{t}\right\}$ is said to be a local martingale if there exists an increasing sequence of stopping times $\left\{\tau_{n}\right\}$ such that $\tau_{n} \uparrow \infty$

* Received by the editors September 15, 1970, and in revised form January 20, 1971.

$\dagger$ Department of Electrical Engineering and Computer Sciences and the Electronics Research Laboratory, University of California, Berkeley, California 94720. This work was supported by the United States Army Research Office, Durham, under Contract DAHC04-67-0046 and the National Science Foundation under Grant GK-1065X. 
almost surely and $\left\{X_{\min \left(t, \tau_{n}\right)}, \mathscr{A}_{t}\right\}$ is a second order martingale. Let $t \wedge s$ denote $\min (t, s)$ and set

$$
X_{n, t}=X_{t \wedge \tau_{n}}
$$

Kunita and Watanabe have proved [2] that if $\left\{X_{t}, \mathscr{A}_{t}\right\}$ is a sample-continuous local martingale, then there exists a sample-continuous increasing process $\langle X\rangle_{t}$ such that

$$
\langle X\rangle_{t \wedge \tau_{n}}=\left\langle X_{n}\right\rangle_{t}
$$

Of course, now we need not have $E\langle X\rangle_{t}<\infty$.

If $\left\{W_{t}, \mathscr{A}_{t}\right\}$ is a sample-continuous second order martingale such that for $t>s$,

$$
E^{\mathscr{A}_{s}}\left(W_{t}-W_{s}\right)^{2}=t-s
$$

then $W$ is necessarily a Brownian motion and for each $s,\left\{W_{t}-W_{s}, t \geqq s\right\}$ is independent of $\mathscr{A}_{s}\left[3\right.$, p. 384]. We describe this situation by saying that $\left\{W_{t}, \mathscr{A}_{t}\right\}$ is a Brownian motion. Let $\left\{W_{t}, \mathscr{A}_{t}\right\}$ be a Brownian motion and let $\left\{\phi_{t}, t \geqq 0\right\}$ be a measurable process adapted to $\left\{\mathscr{A}_{t}\right\}$ such that

$$
\int_{0}^{t} E \phi_{s}^{2} d s<\infty
$$

for each $t$. The stochastic integral $\int_{0}^{t} \phi_{s} d W_{s}$ is well-defined as the quadratic limit of a sequence of sums $\sum_{v} \phi_{t_{v}^{(n)}}\left[W_{t_{v}^{(n)}+1}-W_{t_{v}^{(n)}}\right]$, where $\left\{t_{v}^{(n)}\right\}$ is a sequence of partitions of $[0, t]$ such that

$$
\max _{v}\left(t_{v+1}^{(n)}-t_{v}^{(n)}\right) \underset{n \rightarrow \infty}{\longrightarrow} 0
$$

If we define

$$
X_{t}=\int_{0}^{t} \phi_{s} d W_{s}
$$

and choose a separable version for $X$, then $\left\{X_{t}, \mathscr{A}_{t}\right\}$ is a second order samplecontinuous martingale, with

$$
E^{\mathscr{A}_{s}}\left(X_{t}-X_{s}\right)^{2}=\int_{s}^{t} E^{\mathscr{A}_{s}} \phi_{\tau}^{2} d \tau, \quad 0 \leqq s \leqq t .
$$

If, instead of (4), $\phi$ merely satisfies

$$
\int_{0}^{t} \phi_{s}^{2} d s<\infty, \quad \text { a.s. }
$$

then $\int_{0}^{t} \phi_{s} d W_{s}$ can be defined as follows: Let $\tau_{n}(\omega)$ be defined by

$$
\tau_{n}(\omega)=\left\{\begin{array}{l}
\inf \left\{t: \int_{0}^{t} \phi_{s}^{2}(\omega) d s \geqq n\right\} \\
\infty \quad \text { if } \int_{0}^{t} \phi_{s}^{2}(\omega) d s<n \text { for all } t,
\end{array}\right.
$$


and set

$$
\phi_{n, s}(\omega)= \begin{cases}\phi_{s}(\omega), & s \leqq \tau_{n}(\omega), \\ 0, & s>\tau_{n}(\omega)\end{cases}
$$

For each $n, \int_{0}^{t} \phi_{n, s} d W_{s}$ is well-defined. It can be shown that $\int_{0}^{t} \phi_{n, s} d W_{s}$ converges in probability as $n \rightarrow \infty$, and we define $\int_{0}^{t} \phi_{s} d W_{s}$ as this limit. Now, the process $X_{t}=\int_{0}^{t} \phi_{s} d W_{s}$ need no longer be second order or a martingale, but it is still sample-continuous if a separable version is chosen. Moreover, for each $n$, $\left\{X_{t \wedge \tau_{n}}, \mathscr{A}_{t}\right\}$ is a second order martingale. By definition, $X$ is a local martingale.

If $X$ is a stochastic integral of the form

$$
X_{t}=X_{0}+\int_{0}^{t} \phi_{s} d W_{s}
$$

and $f$ is a twice continuously differentiable function of a real variable, then Ito's differentiation formula [4] yields

$$
f\left(X_{t}\right)=f\left(X_{0}\right)+\int_{0}^{t} f^{\prime}\left(X_{s}\right) \phi_{s} d W_{s}+\frac{1}{2} \int_{0}^{t} f^{\prime \prime}\left(X_{s}\right) \phi_{s}^{2} d s .
$$

In particular,

$$
X_{t}^{2}-X_{0}^{2}=2 \int_{0}^{t} X_{s} \phi_{s} d W_{s}+\int_{0}^{t} \phi_{s}^{2} d s
$$

We can now identify $X_{0}^{2}+2 \int_{0}^{t} X_{s} \phi_{s} d W_{s}$ as the local martingale term in the decomposition (1) of $X_{t}^{2}$, and thus the increasing process is given by

$$
\langle X\rangle_{t}=\int_{0}^{t} \phi_{s}^{2} d s .
$$

If $X$ is of the form (10), then we can define the stochastic integral $\int_{0}^{t} \psi_{s} d X_{s}$ by

$$
\int_{0}^{t} \psi_{s} d X_{s}=\int_{0}^{t} \psi_{s} \phi_{s} d W_{s}
$$

provided that $\int_{0}^{t} \psi_{s}^{2} \phi_{s}^{2} d s<\infty$ almost surely. If $X$ is a local martingale, not necessarily of the form (10), the stochastic integral $\int_{0}^{t} \phi_{s} d X_{s}$ can still be defined, provided that $\int_{0}^{t} \phi_{s}^{2} d\langle X\rangle_{s}<\infty$ almost surely [2]. Finally, if $X=Y+Z$ where $Y$ is a local martingale and $\left\{Z_{t}, t \geqq 0\right\}$ is a process with sample functions almost surely of bounded variation, then we can define $\int_{0}^{t} \psi_{s} d X_{s}$ by

$$
\int_{0}^{t} \psi_{s} d X_{s}=\int_{0}^{t} \psi_{s} d Y_{s}+\int_{0}^{t} \psi_{s} d Z_{s}
$$

provided that the first integral exists as a stochastic integral and the second as a Stieltjes integral. For the case where $Y$ and $Z$ are sample continuous and $f$ is a 
twice continuously differentiable function, Kunita and Watanabe has extended Ito's differentiation rule to read

$$
f\left(X_{t}\right)=f\left(X_{0}\right)+\int_{0}^{t} f^{\prime}\left(X_{s}\right) d X_{s}+\frac{1}{2} \int_{0}^{t} f^{\prime \prime}\left(X_{s}\right) d\langle Y\rangle_{s} .
$$

In particular, we note that

$$
X_{t}^{2}-X_{0}^{2}-2 \int_{0}^{t} X_{s} d X_{s}=\langle Y\rangle_{t}
$$

is independent of $Z$ and serves to define $\langle X\rangle_{t}$.

3. Representation of martingales. Not every sample-continuous second order martingale can be represented as a stochastic integral in the form of (10). It is clear from (13) that for such a representation to be possible the increasing process $\langle X\rangle(\omega, t)$ must be an absolutely continuous function of $t$ (w.r.t. the Lebesgue measure) for almost all $\omega$. As Fisk has observed [5], this condition is also sufficient by virtue of a theorem of Doob [3, p. 449], but it may be necessary to enlarge the underlying probability space by the adjunction of a Brownian motion. Specifically, Doob proved the following theorem.

THEOREM 3.1 (Doob). Let $\left\{X_{t}, \mathscr{A}_{t}, 0 \leqq t \leqq T\right\}$ be a sample-continuous second order martingale. Suppose that there exists a nonnegative measureable process $\left\{\psi_{t}, 0 \leqq t \leqq T\right\}$ adapted to $\left\{\mathscr{A}_{t}\right\}$ such that for $t>s$,

$$
E^{\mathscr{A}_{s}}\left(X_{t}-X_{s}\right)^{2}=\int_{s}^{t} E^{\mathscr{A}_{s}} \psi_{\tau} d \tau
$$

If the set $\{(\omega, t): \psi(\omega, t)=0\}$ has zero $d \mathscr{P} d t$ measure, then there exists a Brownian motion $\left\{W_{t}, \mathscr{A}_{t}, 0 \leqq t \leqq T\right\}$ such that

$$
X_{t}=X_{0}+\int_{0}^{t} \psi_{s}^{1 / 2} d W_{s}
$$

with probability 1 . Without the hypothesis that $\psi$ vanishes almost nowhere, representation (15) is still valid with the adjunction of a Brownian motion to the probability space.

The condition that $\langle X\rangle$ be almost surely continuous with respect to the Lebesgue measure is both somewhat stringent and difficult to verify. Perhaps, it is more natural to consider representations of a more general form

$$
X_{t}(\omega)=X_{0}(\omega)+\int_{0}^{t} \phi_{s}(\omega) d W_{F(s)}(\omega)
$$

where $W$ is a Brownian motion and $F$ is a continuous increasing function.

THEOREM 3.2. Let $\left\{X_{t}, \mathscr{A}_{t}, 0 \leqq t \leqq T\right\}$ be a sample-continuous local martingale. We assume that $\left\{\mathscr{A}_{t}\right\}$ is right-continuous (i.e. $\bigcap_{s>t} \mathscr{A}_{s}=\mathscr{A}_{t}$ ) and each $\mathscr{A}_{t}$ is completed. A representation of the form (16) exists if and only if $\langle X\rangle$ is absolutely continuous with respect to $F$ with probability 1. 
Proof. We only need to prove the theorem for the case $F(t)=t$, because $\left\{X_{t}, \mathscr{A}_{t}\right\}$ can be transformed into a sample-continuous local martingale $\left\{\tilde{X}_{t}, \tilde{\mathscr{A}}_{t}\right\}$ with $E \widetilde{X}_{t}^{2}=t$ by defining

$$
\begin{aligned}
F^{-1}(t) & =\inf \{s: F(s)=t\}, \\
\tilde{X}_{t} & =X_{F^{-1}(t)}, \\
\tilde{\mathscr{A}}_{t} & =\mathscr{A}_{F^{-1}(t)} .
\end{aligned}
$$

Even though $F^{-1}$ may be discontinuous, $\left\{\tilde{\mathscr{A}}_{t}\right\}$ is right-continuous and $\tilde{X}$ is still sample-continuous, because $F(t)=F(s)$ implies $X_{t}=X_{s}$ almost surely. Since $X_{t}=\tilde{X}_{F(t)}$ with probability 1 , a representation

$$
\tilde{X}_{t}=\tilde{X}_{0}+\int_{0}^{t} \tilde{\phi}_{s} d W_{s}
$$

where $\left\{W_{t}, \tilde{\mathscr{A}}_{t}\right\}$ is a Brownian motion, implies a representation

$$
\begin{aligned}
X_{t} & =\tilde{X}_{F(t)}=X_{0}+\int_{0}^{F(t)} \tilde{\phi}_{s} d W_{s} \\
& =X_{0}+\int_{0}^{t} \phi_{s} d W_{F(s)},
\end{aligned}
$$

which is just (16).

To prove Theorem 3.2 for the case $F(t)=t$, we first note that necessity follows from (13). To prove sufficiency, we assume that $\langle X\rangle$ is absolutely continuous with probability 1 and write

$$
\langle X\rangle_{t}(\omega)=\int_{0}^{t} \psi_{s}(\omega) d s
$$

where $\psi$ can always be chosen to be a measurable process because $\langle X\rangle$ is a measurable process. For each $t, \psi_{t}$ is measurable with respect to $\bigcap_{s>t} \mathscr{A}_{s}$, which is equal to $\mathscr{A}_{t}$ by assumption.

We now follow Doob [3, p. 449] and define

$$
g_{s}(\omega)= \begin{cases}\psi_{s}^{-1 / 2}(\omega) & \text { if } \psi_{s}(\omega)>0, \\ 0 & \text { if } \psi_{s}(\omega)=0 .\end{cases}
$$

Since $\int_{0}^{t} g_{s}^{2} d\langle X\rangle_{s} \leqq t<\infty$, the integral $\int_{0}^{t} g_{s} d X_{s}=W_{t}$ is well-defined as a local martingale. If $\psi_{s}(\omega)>0$ for almost all $(\omega, s)$, then $\langle W\rangle_{t}=t$ and it follows from Theorem 2.3 of [2] that $W$ is a Brownian motion. If not, we adjoin an independent Brownian motion $B$ to the underlying probability space and define

$$
W_{t}=\int_{0}^{t} g_{s} d X_{s}+\int_{0}^{t}\left[1-g_{s} \sqrt{\psi_{s}}\right] d B_{s} .
$$

Then, $W$ is a Brownian motion. In either case, we have

$$
X_{t}=X_{0}+\int_{0}^{t} \sqrt{\psi_{s}} d W_{s}
$$

and the proof is complete. 
Theorem 3.2 is basically the same as Theorem 2.1 of [5], except for the introduction of the increasing function $F$ and the generalization to local martingales. We now come to the main result of the section, namely, a sufficient condition for the representation (16) that can be verified in terms of two-dimensional distributions of a martingale $X$.

TheOREM 3.3. Let $\left\{X_{t}, \mathscr{A}_{t}, 0 \leqq t \leqq T\right\}$ be a sample-continuous second order martingale and let $F(t)=E\left(X_{t}-X_{0}\right)^{2}=E\langle X\rangle_{t}$. Suppose that there exist finite positive constants $\alpha$ and $\beta$ such that

$$
\sup _{0<F(t)-F(s) \leqq \beta} \frac{E\left|X_{t}-X_{s}\right|^{2+2 \alpha}}{[F(t)-F(s)]^{1+\alpha}}<\infty .
$$

Then $\langle X\rangle$ is almost surely absolutely continuous with respect to the F-measure, and $X$ has a representation of the form of (16).

Proof. By virtue of the Lebesgue decomposition, we can always write

$$
\langle X\rangle_{t}(\omega)=\int_{0}^{t} \psi_{s}(\omega) d F(s)+\mu_{t}(\omega)
$$

where $\mu$ is almost surely singular with respect to $F$. Now, $E \psi_{s}=1$ implies

$$
E \mu_{t}=-\int_{0}^{t} d F(t)+E\langle X\rangle_{t}=0,
$$

which in turn implies that $\mu \equiv 0$ almost surely since $\mu$ is nonnegative and samplecontinuous. Therefore, we only need to prove that (19) implies $E \psi_{s}=1,0 \leqq s \leqq T$.

Let $T_{n}=\left\{t_{v}^{(n)}, v=0,1, \cdots, n\right\}$ be a sequence of nested (i.e., $T_{n+1} \supset T_{n}$ ) partitions of the interval $[0, T]$ such that

$$
\max _{v}\left[F\left(t_{v+1}^{(n)}\right)-F\left(t_{v}^{(n)}\right)\right] \underset{n \rightarrow \infty}{\longrightarrow} 0 .
$$

Define $\psi_{n, t}, 0 \leqq t \leqq T$, as follows :

$$
\psi_{n, t}=\frac{\langle X\rangle_{t_{v}^{(n)}}-\langle X\rangle_{t_{v}^{(n)}}}{F\left(t_{v+1}^{(n)}\right)-F\left(t_{v}^{(n)}\right)}, \quad t_{v}^{(n)} \leqq t<t_{v+1}^{(n)} .
$$

It is well known (see, e.g., [3, pp. 346-347]) that for each $\omega, \psi_{n . t}$ converges for almost all $t$ ( $F$-measure) to the Radon-Nikodym derivative of the absolutely continuous component of $\langle X\rangle$ with respect to $F$. That is, $\psi_{n, t} \rightarrow \psi_{t}$ for almost all $(\omega, t)$. Since it is obvious that $E \psi_{n, t}=1$, the desired result $E \psi_{t}=1$ will follow if for each $t$, $\left\{\psi_{n, t}\right\}$ is a uniformly integrable family of random variables.

Now, it is known [6] that for any $p>1 / 2$ there exists a constant $\kappa_{p}$ such that

$$
E\left|\langle X\rangle_{t}-\langle X\rangle_{s}\right|^{p} \leqq \kappa_{p} E\left|X_{t}-X_{s}\right|^{2 p} \text {. }
$$

Therefore, if we let $N$ be the smallest $n$ such that

$$
\max _{v}\left[F\left(t_{v+1}^{(n)}\right)-F\left(t_{v}^{(n)}\right)\right] \leqq \beta,
$$

then

$$
\sup _{n \geqq N} E \psi_{n, t}^{1+\alpha} \leqq \kappa_{1+\alpha} \sup _{0<F(t)-F(s) \leqq \beta}\left\{\frac{E\left|X_{t}-X_{s}\right|^{2+2 \alpha}}{[F(t)-F(s)]^{1+\alpha}}\right\}<\infty,
$$


so that $\left\{\psi_{n, t}\right\}$ is a uniformly integrable family of random variables. This, together with Theorem 3.2, completes the proof.

Theorem 3.2 is reminiscent of Kolmogorov's condition for sample continuity and has similar advantages, the primary one being that it can be verified in terms of the two-dimensional distributions of $X$.

4. Quadratic variation. Let $T_{n}=\left\{t_{v}^{(n)}\right\}$ be a nested sequence of partitions of $[0, T]$ such that $\max _{v}\left(t_{v}^{(n)}-t_{v+1}^{(n)}\right) \underset{n \rightarrow \infty}{\longrightarrow} 0$. Let $X$ be a sample-continuous second order martingale, and let $t \wedge s$ denote $\min (t, s)$. Fisk [5] has shown that the sequence of sums

$$
Q_{n}(X, t)=\sum_{v}\left[X_{t \wedge t_{v}^{(n+1}}-X_{t \wedge t_{v}^{(n)}}\right]^{2}
$$

converges to $\langle X\rangle_{t}$ in $L^{1}$-mean, i.e.,

$$
E\left|Q_{n}(X, t)-\langle X\rangle_{t}\right| \underset{n \rightarrow \infty}{\longrightarrow} 0 .
$$

For this reason, $\langle X\rangle_{t}$ is said to be the quadratic variation of $X$ on $[0, t]$. Now, suppose that $\left\{Z_{t}, 0 \leqq t \leqq T\right\}$ is a sample-continuous process, the sample functions of which are almost surely of bounded variation. Then there exists an almost surely finite random variable $A$ such that

$$
\sup _{n} \sum_{v}\left|Z_{t_{v+1}^{(n)}}-Z_{t_{v}^{(n)}}\right| \leqq A .
$$

Therefore,

$$
\begin{aligned}
Q_{n}(Z, T)=\sum_{v}\left|Z_{t_{v+1}^{(n)}}-Z_{t_{v}^{(n)}}\right|^{2} \leqq A \max _{v}\left|Z_{t_{v+1}^{(n)}}-Z_{t_{v}^{(n)}}\right| \\
\stackrel{\text { a.s. }}{\underset{n \rightarrow \infty}{\longrightarrow}} 0
\end{aligned}
$$

so that $Z$ has zero quadratic variation on $[0, T]$.

THEOREM 4.1. Let $\left\{\mathscr{A}_{t}\right\}$ and $\left\{\tilde{\mathscr{A}}_{t}\right\}$ be two increasing families of $\sigma$-algebras and let $\left\{X_{t}, \mathscr{A}_{t}\right\}$ and $\left\{\tilde{X}_{t}, \tilde{\mathscr{A}}_{t}\right\}$ be sample-continuous local martingales. If $X+\tilde{X}$ or $X-\tilde{X}$ is of bounded variation, then $\langle X\rangle_{t}=\langle\tilde{X}\rangle_{t}$ almost surely for every $t$.

Proof. First, suppose that $X$ and $\tilde{X}$ are second order martingales. Let $B$ denote the process of bounded variation given by $X-\tilde{X}$ or $X+\tilde{X}$. From the inequality

we have

$$
\left(\sqrt{\sum_{k} a_{k}^{2}}-\sqrt{\sum_{k} b_{k}^{2}}\right)^{2} \leqq \sum_{k}\left(a_{k} \pm b_{k}\right)^{2} \leqq\left(\sqrt{\sum_{k} a_{k}^{2}}+\sqrt{\sum_{k} b_{k}^{2}}\right)^{2},
$$

$$
\left(\sqrt{Q_{n}(\tilde{X}, t)}-\sqrt{Q_{n}(B, t)}\right)^{2} \leqq Q_{n}(X, t) \leqq\left(\sqrt{Q_{n}(\tilde{X}, t)}+\sqrt{Q_{n}(B, t)}\right)^{2} .
$$

Since $Q_{n}(\tilde{X}, t), Q_{n}(X, t)$ and $Q_{n}(B, t)$ converge respectively to $\langle\tilde{X}\rangle_{t},\langle X\rangle_{t}$ and 0 , we have $\langle X\rangle_{t}=\langle\tilde{X}\rangle_{t}$ almost surely for all $t \in[0, T]$.

If $X$ and $\tilde{X}$ are local martingales, then there exist sequences of stopping times $\left\{\tau_{m}\right\}$ and $\left\{\tilde{\tau}_{m}\right\}$ increasing to $\infty$, so that $X_{m, t}=X_{\tau_{m} \wedge t}$ and $\tilde{X}_{m, t}=\tilde{X}_{\tau_{m} \wedge t}$ are second order martingales for each $m$. Let

$$
\Omega_{m, t}=\left\{\omega: t<\tau_{m} \wedge \tilde{\tau}_{m}\right\}
$$


Since $\tau_{m} \uparrow \infty$ and $\widetilde{B}_{m} \uparrow \infty$, we have $\Omega_{m, t} \uparrow \Omega$. For $\omega \in \Omega_{m, t}$, we have

$$
\begin{aligned}
& X_{m, s}(\omega)=X_{s}(\omega), \quad 0 \leqq s \leqq t, \\
& \tilde{X}_{m, s}(\omega)=\tilde{X}_{s}(\omega), \quad 0 \leqq s \leqq t .
\end{aligned}
$$

Therefore, for $\omega \in \Omega_{m, t}$, either $X_{m}(\omega)+\tilde{X}_{m}(\omega)$ or $X_{m}(\omega)-\tilde{X}_{m}(\omega)$ is of bounded variation on $[0, t]$, and it follows from the same inequality as before that

$$
\left\langle X_{m}\right\rangle_{t}(\omega)=\left\langle\tilde{X}_{m}\right\rangle_{t}(\omega), \quad \omega \in \Omega_{m, t},
$$

or

$$
\langle X\rangle_{t}(\omega)=\langle\tilde{X}\rangle_{t}(\omega), \quad \omega \in \Omega_{m, t} .
$$

The proof is completed by letting $m \uparrow \infty$.

We should note that in Theorem 4.1 we do not assume that $X$ and $\tilde{X}$ are local martingales with respect to the same family of $\sigma$-algebras. If they are, then $X+\tilde{X}$ and $X-\tilde{X}$ are both local martingales. If one of them is also of bounded variation, say $X+X$, then $X_{t}+\tilde{X}_{t}=X_{0}+\tilde{X}_{0}$ with probability 1 for all $t \in[0, T]$, in which case the result of Theorem 4.1 trivially follows. The more interesting cases arise when neither $X+\tilde{X}$ nor $X-\tilde{X}$ is a local martingale.

An interesting application of Theorem 4.1 is in connection with quasi-martingales [7]. A process $\left\{X_{t}, 0 \leqq t \leqq T\right\}$ is said to be a quasi-martingale with respect to $\left\{\mathscr{A}_{t}\right\}$ if there exist $\left\{B_{t}, 0 \leqq t \leqq T\right\}$ and $\left\{M_{t}, 0 \leqq t \leqq T\right\}$ both adapted to $\left\{\mathscr{A}_{t}\right\}$ such that $X=M+B, B$ is of bounded variation, and $\left\{M_{t}, \mathscr{A}_{t}\right\}$ is a martingale. We shall be interested only in those cases where both $M$ and $B$ are samplecontinuous and where the total variation of $B$ has a finite expectation. Under these assumptions, if $\left\{X_{t}, \mathscr{A}_{t}\right\}$ is a quasi-martingale, then $\left\{X_{t}, \mathscr{A}_{x t}\right\}$ is always a quasi-martingale, where $\mathscr{A}_{x t}$ denotes the $\sigma$-algebra generated by $\left\{X_{s}, 0 \leqq s \leqq t\right\}$. More generally, let $\left\{\mathscr{A}_{t}\right\}$ be any increasing family of $\sigma$-algebras such that $\mathscr{A}_{x t}$ $\subseteq \tilde{\mathscr{A}}_{t} \subseteq \mathscr{A}_{t}$ for every $t$. Then, $\left\{X_{t}, \tilde{\mathscr{A}}_{t}\right\}$ is a quasi-martingale. ${ }^{1}$ That is, there exist $\widetilde{B}$ and $\tilde{M}$ adapted to $\left\{\mathscr{A}_{t}\right\}$ such that $B$ is of bounded variation. $\left\{\tilde{M}_{t}, \tilde{\mathscr{A}}_{t}\right\}$ is a martingale, both $\tilde{M}$ and $\widetilde{B}$ are sample-continuous, and the total variation of $\widetilde{B}$ has finite expectation. It follows from Theorem 4.1 that $\langle M\rangle_{t}=\langle\tilde{M}\rangle_{t}$ for every $t$.

An important class of quasi-martingales is made up of Ito processes, which are processes having the representation

$$
X_{t}=X_{0}+\int_{0}^{t} \psi_{s} d s+\int_{0}^{t} \phi_{s} d W_{s}, \quad 0 \leqq t \leqq T
$$

where $\psi$ and $\phi$ are measurable processes adapted to an increasing family of $\sigma$-algebras $\left\{\mathscr{A}_{t}\right\},\left\{W_{t}, \mathscr{A}_{t}\right\}$ is a Brownian motion, and $X_{0}$ is $\mathscr{A}_{0}$-measurable. In addition, we assume

$$
\begin{aligned}
& \int_{0}^{T} E\left|\psi_{s}\right| d s<\infty, \\
& \int_{0}^{T} E \phi_{s}^{2} d s<\infty .
\end{aligned}
$$

\footnotetext{
${ }^{1}$ This fact is easily proved by verifying the two conditions of Theorem 3.3 in [7].
} 
It is clear that $\left\{X_{t}, \mathscr{A}_{t}\right\}$ is a quasi-martingale. Thus, $\left\{X_{t}, \mathscr{A}_{x t}\right\}$ is also a quasimartingale and the representation of $X$ as a quasi-martingale with respect to $\left\{\mathscr{A}_{x t}\right\}$ is given in the following theorem.

THEOREM 4.2. Let $X_{t}, 0 \leqq t \leqq T$, be an Ito process satisfying (24), (25) and

$$
\int_{0}^{T} \phi_{s}^{2} d s<\infty \quad \text { almost surely. }
$$

Then there exists a representation of $X$ in the form

$$
X_{t}=X_{0}+\int_{0}^{t} E^{\tilde{A}_{s}} \psi_{s} d s+\int_{0}^{t}\left|\psi_{s}\right| d \tilde{W}_{s}
$$

where $\left\{\tilde{\mathscr{A}}_{t}\right\}$ is any increasing family of $\sigma$-algebras satisfying

$$
\mathscr{A}_{x t} \subseteq \tilde{\mathscr{A}}_{t} \subseteq \mathscr{A}_{t} \text { for each } t
$$

and $\tilde{W}$ is a Brownian motion.

Remark. If we take $\tilde{\mathscr{A}}_{t}=\mathscr{A}_{x t}$, then we have

$$
X_{t}=X_{0}+\int_{0}^{t} E^{\mathscr{A}_{x s}} \psi_{s} d s+\int_{0}^{t}\left|\phi_{s}\right| d \tilde{W}_{s}
$$

where both integrands $E^{\mathscr{A} x s} \psi_{s}$ and $\left|\phi_{s}\right|$ are $\mathscr{A}_{x s}$-measurable functions for almost all $s$. The latter stems from the fact that

$$
\left|\phi_{t}\right|=\left[\frac{d}{d t}\langle X\rangle_{t}\right]^{1 / 2},
$$

where $d / d t$ stands for the Radon-Nikodym derivative and $\langle X\rangle_{t}$ is defined by

$$
\langle X\rangle_{t}=X_{t}^{2}-X_{0}^{2}-2 \int_{0}^{t} X_{s} d X_{s}
$$

Proof. First, suppose that, in place of (27), the stronger condition (26) is satisfied. Then, $\left\{X_{t}, \tilde{\mathscr{A}}_{t}\right\}$ is a quasi-martingale and we can write

$$
X_{t}=X_{0}+\widetilde{B}_{t}+\tilde{\mathscr{A}}_{t}, \quad 0 \leqq t \leqq T,
$$

where $\widetilde{B}$ is of bounded variation and $\left\{\tilde{M}_{t}, \tilde{\mathscr{A}}_{t}\right\}$ is a martingale. Since

$$
\tilde{M}_{t}-\int_{0}^{t} \phi_{s} d W_{s}=\int_{0}^{t} \psi_{s} d s-\tilde{B}_{t}
$$

and $M_{t}=\int_{0}^{t} \phi_{s} d W_{s}$ is a martingale under condition (26), we have from Theorem 4.1:

$$
\langle\tilde{M}\rangle_{t}=\langle M\rangle_{t}=\int_{0}^{t} \phi_{s}^{2} d s, \quad 0 \leqq t \leqq T ;
$$

Theorem 3.1 yields the representation

$$
\langle\tilde{M}\rangle_{t}=\int_{0}^{t}\left|\phi_{s}\right| d \tilde{W}_{s}
$$

where $\tilde{W}$ is a Brownian motion. 
To get an integral representation for $\widetilde{B}$, let $T_{n}=\left\{t_{v}^{(n)}\right\}, n=1,2, \cdots$, be a nested sequence of partitions of $[0, T]$ such that $\max _{v}\left(t_{v+1}^{(n)}-t_{v}^{(n)}\right) \underset{n \rightarrow \infty}{\longrightarrow} 0$. Define

$$
\widetilde{B}_{n, t}=\sum_{v} E\left[X_{t \wedge t_{v}^{(n+1}}-X_{\left.t \wedge t_{v}^{(n)}\right)} \tilde{\mathscr{A}}_{t_{v}^{(n)}}\right] .
$$

Fisk [7] has shown that

$$
E\left|\widetilde{B}_{n, t}-\widetilde{B}_{t}\right| \underset{n \rightarrow \infty}{\longrightarrow} 0 .
$$

From (24) we get

$$
E\left[X_{t_{v}^{(n+1}}-X_{t_{v}^{(n)}} \mid \tilde{A}_{t_{v}^{(n)}}\right]=\int_{t_{v}^{(n)}}^{t_{v}^{(n+1}} E\left(\psi_{s} \mid \tilde{A}_{t_{v}^{(n)}}\right) d s
$$

If we denote $f_{s}=E^{\tilde{\mathscr{A}}_{s}} \psi_{s}$, then

$$
\begin{aligned}
& \sup _{0 \leqq t \leqq T} E\left|\int_{0}^{t} E^{\tilde{A}_{s}} \psi_{s} d s-\tilde{B}_{t}\right| \\
& \leqq \lim _{n \rightarrow \infty}\left\{E\left|\sum_{v} \int_{t_{v}^{(n)}}^{t_{v}^{(n)}}\left[f_{s}-f_{t_{v}^{(n)}}\right] d s\right|\right. \\
& \left.\quad+E\left|\sum_{v} \int_{T_{v}^{(n)}}^{t_{v}^{(n)}+1} E\left[\psi_{s}-\psi_{\left.t_{v}^{(n)}\right)} \tilde{A}_{t_{v}^{(n)}}\right] d s\right|\right\} \\
& \leqq \lim _{n \rightarrow \infty} E \sum_{v} \int_{t_{v}^{(n)}}^{t_{v}^{(n)}}\left[\left|f_{s}-f_{t_{v}^{(n)}}\right|+\left|\psi_{s}-\psi_{t_{v}^{(n)}}\right|\right] d s .
\end{aligned}
$$

For a suitable sequence of partitions $\left\{t_{v}^{(n)}\right\}$, the last limit can always be made zero (Doob [3, pp. 63-65]). Since the first expression is independent of $\left\{t_{v}^{(n)}\right\}$, we must have

$$
\sup _{0 \leqq t \leqq T} E\left|\int_{0}^{t} E^{\tilde{a} \tilde{s}_{s}} \psi_{s} d s-\tilde{B}_{t}\right|=0 .
$$

If $\phi$ satisfies (27) and not (26), we define $\left\{\tau_{n}\right\}$ by (8). Since $\langle X\rangle_{t}=\int_{0}^{t} \psi_{s}^{2} d s$, $\left\{\tau_{n}\right\}$ is a sequence of stopping times not only for $\left\{\mathscr{A}_{t}\right\}$ but also for $\left\{\mathscr{A}_{t}\right\}$. Therefore, for each $n,\left\{X_{t \wedge \tau_{n}}, \tilde{\mathscr{A}}_{t}\right\}$ is a quasi-martingale with a representation

$$
X_{t \wedge \tau_{n}}=X_{0}+\int_{0}^{t \wedge \tau_{n}} E^{\tilde{\mathscr{A}}_{s}} \psi_{s} d s+\tilde{M}_{t \wedge \tau_{n}} .
$$

Hence, $\tilde{M}_{t}=X_{t}-X_{0}-\int_{0}^{t} E^{\tilde{a_{s}}} \psi_{s} d s$ is a local martingale. It follows from Theorem 4.1 and (24) that

$$
\langle\tilde{M}\rangle_{t}=\int_{0}^{t} \phi_{s}^{2} d s,
$$

and the proof is completed by using Theorem 3.2 .

Theorem 4.2 can be generalized to a vector Ito process practically without change. Let $X$ be an $n$-vector-valued process satisfying

$$
X_{t}=X_{0}+\int_{0}^{t} \psi_{s} d s+\int_{0}^{t} \phi_{s} d W_{s},
$$


where $W$ is an $m$-vector process, the components of which are independent Brownian motions and $\phi$ is an $n \times m$ matrix. Instead of (25) and (27), we now assume

$$
\int_{0}^{T} E\left\|\psi_{s}\right\| d s<\infty
$$

and

$$
\int_{0}^{T}\left\|\phi_{s}\right\|^{2} d s<\infty \quad \text { almost surely, }
$$

where $\|\cdot\|$ denotes the Euclidean norm.

Let $\alpha$ be an $n$-vector and let prime denote transpose. For every $\alpha \in R^{n},\left\{\alpha^{\prime} X_{t}\right\}$ is a scalar-valued Ito process of the form (24), and we can write

$$
\alpha^{\prime} X_{t}=\int_{0}^{t} \alpha^{\prime} E^{\tilde{\tilde{A}_{s}}} \psi_{s} d s+M_{\alpha, t},
$$

where $\left\{M_{\alpha, t} \tilde{\mathscr{A}}_{t}\right\}$ is a local martingale for any $\left\{\tilde{\mathscr{A}}_{t}\right\}$ satisfying (29) and

$$
\left\langle M_{\alpha}\right\rangle_{t}=\int_{0}^{t} \alpha^{\prime} \phi_{s} \phi_{s}^{\prime} \alpha d s
$$

It follows that $\int_{0}^{t} \phi_{s} \phi_{s}^{\prime} d s$ is $\mathscr{A}_{x t}$-measurable for every $t$, and the positive semidefinite matrix $\phi_{s} \phi_{s}^{\prime}$ is $\mathscr{A}_{x s}$-measurable for almost all $s$. Let $\phi_{s} \phi_{s}^{\prime}$ be diagonalized so that

$$
\phi_{s} \phi_{s}^{\prime}=A_{s} \Lambda_{s} A_{s}^{\prime},
$$

where $\Lambda_{s}$ is diagonal and the orthogonal matrix $A_{s}$ is $\mathscr{A}_{x t}$-measurable for almost all $s$. Now, if we define

$$
Y_{t}=\int_{0}^{t} A_{s}^{\prime} d X_{s}
$$

and apply Theorem 4.2 to the components of $Y$, we get the following theorem.

THEOREM 4.3. Let $X$ be an n-vector Ito process of the form (32) satisfying (33) and (34). Let $A_{s}$ and $\Lambda_{s}$ be matrices defined by the diagonalization (35). Then, for any increasing family of $\sigma$-algebras satisfying (29), there exists an $n$-vector Brownian motion $\tilde{W}$ so that

$$
X_{t}=X_{0}+\int_{0}^{t} E^{\mathscr{A}_{x s}} \psi_{s} d s+\int_{0}^{t} A_{s} \Lambda_{s}^{1 / 2} d \tilde{W}_{s}
$$

5. Applications. Equation (32) is widely used to model a dynamical system disturbed by Gaussian white noise. Theorem 4.3 has some interesting and surprising consequences for such models. For example, in filtering problems we often interpret $X$ in (32) as the observed process, $\psi$ as the process to be estimated, and the stochastic integral term as the noise. If we identify $\hat{\psi}_{t}=E^{\mathscr{A}_{x t}} \psi_{t}$ as the estimator, then Theorem 4.3 implies that

$$
\int_{0}^{t}\left(\psi_{s}-\hat{\psi}_{s}\right) d s=-\int_{0}^{t} \phi_{s} d W_{s}+\int_{0}^{t} A_{s} \Lambda_{s}^{1 / 2} d \tilde{W}_{s} .
$$


The stochastic integral $-\int_{0}^{t} \phi_{s} d W_{s}$ can always be re-represented as $\int_{0}^{t} A_{\mathrm{s}} \Lambda_{\mathrm{s}}^{1 / 2} d V_{s}$, where $V$ is now an $n$-dimensional Brownian motion, so that

$$
\int_{0}^{t}\left(\psi_{s}-\hat{\psi}_{s}\right) d s=\int_{0}^{t} A_{s} \Lambda_{s}^{1 / 2}\left(d V_{s}+d \tilde{W}_{s}\right)
$$

Formally, the estimation error can be expressed as

$$
\psi_{t}-\hat{\psi}_{t}=A_{t} \Lambda_{t}^{1 / 2}\left(\dot{V}_{t}+\dot{\tilde{W}}_{t}\right)
$$

One may be tempted to say that the estimation error is "white," but that would be misleading. For the special case $\phi(\omega, t)=K$, a constant, we have

$$
\psi_{t}-\hat{\psi}_{t}=A \Lambda^{1 / 2}\left(\dot{V}_{t}+\dot{\tilde{W}}_{t}\right)
$$

which is the sum of Gaussian white noise (albeit dependent) processes. This was originally observed by Wonham [8] in a more limited context and generalized by Kailath [9]. We note that the same observation is true if $\phi \phi^{\prime}$ is a constant even if $\phi$ is not.

Another observation worthy of note is that for almost all $t, \phi_{t} \phi_{t}^{\prime}$ is $\mathscr{A}_{x t^{-}}$ measurable. Thus, for example, if $\psi_{t}$ is a function only of $t$ and $\left\{\phi_{s} \phi_{s}^{\prime}, 0 \leqq s \leqq t\right\}$, then the estimation error is necessarily zero for almost all $t$. Such a result would be difficult to prove outside of the context of quadratic variations. A similar observation can be made with respect to singular detection [10].

Finally, as a simple example of possible applications to control problems, consider a scalar equation

$$
d X_{t}=f\left(X_{0}^{t}, U_{0}^{t}, t\right) d k+U_{t} d W_{t},
$$

where $X$ represents the state and $U$ the control. Suppose that $U_{t} \geqq 0$ for all $t$. Then Theorem 4.2 implies that for any optimization problem whatever, the optimizing control can always be implemented in state feedback form. This means that even if we observe the past of the noise process $W$ and use it in constructing the control, performance cannot be improved. Surely, this is an unexpected result.

Acknowledgment. I am grateful to Professor P. P. Varaiya for many valuable suggestions. In particular, it was at his persuasion and with his help that I extended the result of $\S 4$ to local martingales and to the multidimensional case.

\section{REFERENCES}

[1] P. A. MeyER, A decomposition theorem for supermartingales, Illinois J. Math., 6 (1962), pp. 193205.

[2] H. Kunita and S. Watanabe, On square integrable martingales, Nagoya Math. J., 30 (1967), pp. 209-245.

[3] J. L. Doob, Stochastic Processes, John Wiley, New York, 1953.

[4] K. Iто, On a formula concerning stochastic differentials, Nagoya Math. J., 3 (1951), pp. 55-65.

[5] D. L. FISK, Sample quadratic variation of sample continuous second order martingales, Z. Wahrscheinlichkeitstheorie und Verw. Gebiete, 6 (1966), pp. 273-278.

[6] P. A. Meyer, Une majoration du processus croissant naturel associé à une surmartingale, Seminaire de probabilités, II, Springer-Verlag, Berlin, 1967.

[7] D. L. FISK, Quasi-martingales, Trans. Amer. Math. Soc., 120 (1965), pp. 369-389.

[8] W. M. Wonham, Random differential equations in control theory, Probabilistic Methods in Applied Mathematics, vol. 2, A. T. Bharucha-Reid, ed., Academic Press, New York, 1970. 
[9] T. KaIlath, An innovation approach to least-squares estimation. Part I: Linear filtering in additive white noise, IEEE Trans. Automatic Control, AC-13 (1968), pp. 646-655.

[10] E. Wong AND M. ZAKAI, The oscillation of stochastic integrals, Z. Wahrscheinlichkeitstheorie und Verw. Gebiete, 4 (1965), pp. 103-112. 